\title{
Scinderin-knockdown inhibits proliferation and promotes apoptosis in human breast carcinoma cells
}

\author{
WENJING JIAN ${ }^{1,2}$, XIAOLI ZHANG ${ }^{3}$, JIGUO WANG $^{4}$, YUNLONG LIU ${ }^{2}$, \\ CHUTING HU ${ }^{2}$, XIANMING WANG ${ }^{2}$ and RENBIN LIU ${ }^{1}$
}

\begin{abstract}
${ }^{1}$ Department of Thyroid and Breast Surgery, The Third Affiliated Hospital, Sun Yat-Sen University, Guangzhou, Guangdong 510630; ${ }^{2}$ Department of Thyroid and Breast Surgery, The Second People's Hospital of Shenzhen; ${ }^{3}$ Central Laboratory, The Second People's Hospital of Shenzhen, Shenzhen, Guangdong 518035; ${ }^{4}$ Department of Medical Oncology, Baoan District Traditional Chinese Medicine Hospital, Shenzhen, Guangdong 518133, P.R. China
\end{abstract}

Received December 16, 2017; Accepted May 11, 2018

DOI: $10.3892 / \mathrm{ol} .2018 .9009$

\begin{abstract}
Previous studies have reported that scinderin (SCIN) affects multiple cellular processes, including proliferation, migration and differentiation in cancer. However, the specific role of SCIN in breast cancer (BC) cells is unknown. Immunohistochemistry was used to investigate SCIN expression in $46 \mathrm{BC}$ and 21 mammary fibroadenoma or fibroadenomatoid hyperplasia tissue samples. SCIN expression was ablated in MDA-MB-231 and T-47D cells using lentivirus-mediated small interfering RNA technology. Cell proliferation was tested using Celigo and 3-(4,5-dimethylthiazol-2-yl)-2,5-diphenyltetrazolium bromide assays. Cell apoptosis was analyzed by measuring Caspase 3/7 activity and annexin-V staining. The results of the present study demonstrated that SCIN expression was elevated in BC tissues compared with mammary fibroadenoma or fibroadenomatoid hyperplasia tissues. Specifically, higher SCIN expression was observed in Ki-67-positive BC tissues (78.6\%) compared with Ki-67-negative BC tissues. Furthermore, knockdown of SCIN expression in the $\mathrm{BC}$ cell lines significantly suppressed cell proliferation and induced apoptosis. The data presented in the present study indicate that SCIN serves an important role in the development of breast cancer.
\end{abstract}

\section{Introduction}

Breast cancer (BC) is one of the most common malignancies in women worldwide (1) and accounts for $25 \%$ (1.7 million) of all cancer cases and 15\% $(521,900)$ of all cancer-related mortalities among females, based on data through 2012 (2).

Correspondence to: Dr Wenjing Jian, Department of Thyroid and Breast Surgery, The Third Affiliated Hospital, Sun Yat-Sen University, 600, Tianhe Road, Guangzhou, Guangdong 510630, P.R. China

E-mail: jianwj2007@126.com

Key words: scinderin, proliferation, apoptosis, breast cancer
The percentage of cancer-related mortalities among females in less developed countries is even higher, for example, female breast cancer mortality in Belize was $14.0 \%$, while $29.7 \%$ in the Cayman Islands in 2013 (3). Despite recent advancements in BC diagnoses and treatments, prognosis outcomes remain unsatisfactory due to the highly complex and heterogeneous nature of this disease (2). Thus, identification of novel therapeutic targets is required to develop additional effective treatment strategies.

Scinderin (SCIN), which is also referred to as adseverin, belongs to the gelsolin super family of actin binding proteins $(4,5)$. SCIN was originally identified in chromaffin cells of the adrenal medulla (6), but is typically expressed in a variety of tissues. For instance, high expression of SCIN was observed in the kidneys and intestines (7), but was expressed at lower levels in other tissue types $(6,8)$. Earlier studies indicated that SCIN serves an important role in the regulation of various cellular processes, including proliferation, migration and differentiation. In human prostate cancer and lung carcinoma cells, SCIN suppression inhibited cell proliferation $(9,10)$. Conversely, in megakaryoblastic leukemia, SCIN overexpression inhibited cell proliferation and tumorigenesis via activating the ras-related $\mathrm{C} 3$ botulinum toxin substrate/p21-activated kinase/mammalian mitogen-activated protein kinase kinase, mitogen-activated protein kinase kinase 4/c-jun n-terminal kinase/c-jun, and rapidly accelerated fibrosarcoma kinase/mitogen-activated protein kinase kinase/extracellular signal-regulated kinase signaling pathways (11). In addition, manipulation of SCIN in gastric cancer cells has been linked to increased cell invasion and metastasis in vivo (12), and has also been revealed to be involved in the differentiation of dental pulp cells (13). However, the role of SCIN in BC is largely unknown.

Thus, in the present study, the biological role of SCIN in $\mathrm{BC}$ was investigated by analyzing its expression in $\mathrm{BC}$ tissue samples, as well as in control mammary fibroadenoma or fibroadenomatoid hyperplasia tissue samples. In addition, SCIN expression was ablated in MDA-MB-231 and T-47D BC cells using lentivirus (LV)-mediated short hairpin (sh)RNA technology to understand the functional 
consequences of knocking down SCIN expression. The results of the present study indicate that SCIN expression serves an important role in inhibiting proliferation and promoting apoptosis in $\mathrm{BC}$ cells.

\section{Materials and methods}

Selection of tumor specimens. This study was approved by the Ethics Committee of the Second People's Hospital of Shenzhen (Shenzhen, Guangdong, China), and written consent was obtained from all study participants. A total of 46 paraffin-embedded $\mathrm{BC}$ specimens, with a median age of 47.54 years (range, 28-64 years) and 21 paraffin-embedded control specimens, with a median age of 29.95 years (range, 17-45 years) collected from patients with mammary fibroadenoma or fibroadenomatoid hyperplasia were obtained from the Center for Breast Disease Diagnosis and Treatment at the Second People's Hospital of Shenzhen between January 2017 and June 2017. All patients were women. Among the 46 cases, 36 were invasive ductal carcinoma (IDC), 6 were ductal carcinoma in situ (DCIS) and 4 were mucoid carcinoma. All diagnoses and classifications were made according to the World Health Organization criteria (14). These resected specimens were $2-3 \mathrm{~mm}$ thick, then fixed in $10 \%$ neutral buffered formalin ( $\mathrm{pH}$ 7.4) at room temperature for $12 \mathrm{~h}$, embedded in paraffin, and cut into $4-\mu \mathrm{m}$ sections.

Cell culture. Human BC MDA-MB-231, T-47D and MCF-7 cell lines were obtained from the Cell Bank of the Chinese Academy of Sciences (Shanghai, China). These cells were cultured in Dulbecco's modified Eagle medium (DMEM; Corning Incorporated, Corning, NY, USA) supplemented with $10 \%$ fetal bovine serum at $37^{\circ} \mathrm{C}$ in a humidified atmosphere with $5 \% \mathrm{CO}_{2}$.

Immunohistochemistry (IHC). Selected paraffin-embedded specimens were cut into $4-\mu \mathrm{m}$ sections and stained using the Dako REAL Envision Detection system (Dako; Agilent Technologies, Inc., Santa Clara, CA, USA) per the manufacturer's protocols. $4-\mu \mathrm{m}$ sections were dewaxed by xylene and rehydrated in a graded ethanol series $(100 \%$ ethanol for $5 \mathrm{~min}, 95 \%$ alcohol for $5 \mathrm{~min}, 90 \%$ alcohol for $3 \mathrm{~min}, 80 \%$ alcohol for $3 \mathrm{~min}, 70 \%$ alcohol for $3 \mathrm{~min}$ at room temperature), following washing 3 times with Phosphate Buffer Solution (PBS) for $10 \mathrm{~min}$ at room temperature. Sections were boiled in $0.01 \mathrm{~mol} / \mathrm{l}$ citrate buffer ( $\mathrm{pH}$ 6.0, MVS-0066, Maixin Biotechnology Development Co., Ltd, Fuzhou, China) at $100^{\circ} \mathrm{C}$ for $4 \mathrm{~min}$, then cooled at room temperature for $1 \mathrm{~h}$ for antigen retrieval. The endogenous peroxidase activity was blocked with $3 \% \mathrm{H}_{2} \mathrm{O}_{2}$ for $10 \mathrm{~min}$ at room temperature, followed by washing 3 times with PBS for $10 \mathrm{~min}$ at room temperature. Next, slides were immersed in 5\% goat serum (YJ0130, Yanjing Biological Co., Shanghai, China) for $10 \mathrm{~min}$ at room temperature to block nonspecific binding. Following removal of goat serum, the slides were incubated with primary rabbit anti-SCIN monoclonal antibody (1:800 dilution; cat. no. ab199723, Abcam, Cambridge, MA, USA) and mouse anti-Ki67 monoclonal antibody (1:100 dilution; cat. no. MAB-0129, MXB Bio-company, Fuzhou, China) at $4^{\circ} \mathrm{C}$ overnight. The next day, the slides were re-heated at room temperature for $1 \mathrm{~h}$. Then washed 3 times with PBS for $10 \mathrm{~min}$ in room temperature, the sections were then incubated with poly-horseradish peroxidase (HRP) anti-rabbit/mouse IgG (cat. no PV-9000, Beijing ZSGB-Bio Company, Beijing, China) for $30 \mathrm{~min}$ at room temperature. DAB staining was performed and later semi-quantitative evaluation of the immunohistochemical signal was performed by two pathologists working independently in a blinded fashion with an Olympus BX-51 microscope (Olympus Corporation, Tokyo, Japan) at x200 magnification (15). The staining intensity of SCIN expression was scored as follows: 0 , Negative (no cytoplasmic SCIN expression); 1, weak (1-25\% staining); 2 , moderate (26-50\% staining); 3 , strong (51-75\% staining); and 4 , high (76-100\%) staining. The staining scores were analyzed using the X-tile software program (v3.6.1; Yale University School of Medicine, New Haven, CT, USA), and a score of 2 was designated as the cutoff value (16). Thus, samples having total scores of $\geq 2$ were classified as having high SCIN expression, while scores of $<2$ represented no or low SCIN expression. Samples that exhibited $<14 \% \mathrm{Ki}-67$ (proliferation marker) expression were grouped as Ki-67 negative (17).

SCIN expression in breast cancer cells from GEO. The information about SCIN expression in BC cells was extracted from GEO (https://www.ncbi.nlm.nih.gov/geo/). The term 'breast cancer cells' was entered and searched in the GEO datasets, and eligible and high quality profiling expression data was identified, 'SCIN' gene was then searched in the GDS4296 datasets and for the expression of SCIN in BC cells.

Generation of LV expressing SCIN, shRNA and target cell transfection. Several shRNAs were designed and tested to target the human SCIN gene in preliminary studies (NM_033128). The shRNA sequence (5'-CGAGATGAGCTGACAACAT-3') that optimally ablated SCIN expression was selected for the present study. A random shRNA sequence (5'-TTCTCCGAACGTGTC ACGT-3') was used as a control. These shRNAs were subsequently ligated into the GV115 lentiviral vector with enhanced green fluorescent protein (GFP) cDNA (GeneChem). To generate LV, MDA-MB-231 and T-47D cells were transfected with GV115-SCIN or control shRNA using X-tremeGENE HP DNA Transfection Reagent (cat no. 6366546001, Roche Diagnostics, Basel, Switzerland), along with two helper plasmids, pHelper 1.0 and pHelper 2.0 (GeneChem), per the manufacturer's protocols. At 2 days post-transfection, the supernatant containing packaged LV was collected and filtered through a $0.45-\mu \mathrm{m}$ filter. To infect MDA-MB-231 and T-47D cells, LV particles, at a multiplicity of transfection of 20, were added to the plated cells in 6-well plates and then cultured at $37^{\circ} \mathrm{C}$ in an incubator. A transfection efficiency of $\sim 80 \%$ was observed via GFP expression using fluorescence microscopy following 3 days of LV transfection. Subsequent experimentations were performed $72 \mathrm{~h}$ following transfection.

$R N A$ extraction and reverse transcription-quantitative polymerase chain reaction ( $R T-q P C R)$. Total RNA was extracted from BC MDA-MB-231, T-47D and MCF-7 cell lines respectively using TRIzol reagent (Thermo Fisher Scientific, Inc., Waltham, MA, USA). The cDNA was 
retro-transcribed from isolated RNA using the M-MLV kit (cat no. M1705, Promega Corporation, Madison, WI, USA). The RT-qPCR reaction conditions were at $95^{\circ} \mathrm{C}$ for $15 \mathrm{sec}$, at $95^{\circ} \mathrm{C}$ for $5 \mathrm{sec}$, then at $60^{\circ} \mathrm{C}$ for $30 \mathrm{sec}$, and the amplification was for 45 cycles using the Real-Time PCR Detection system (cat no. MX3000p; Agilent Technologies, Inc.) with SYBR Premix Ex Taq (cat no. DRR041B, Takara Biotechnology Co., Ltd., Dalian, China) according to the manufacturer's protocols. The primer sequences for SCIN gene amplification were as follows: Forward, AGGAAGGTCTGAACTAAT and reverse, CTGTTACTTATGTCTGCTAT. The primer sequences for GAPDH amplification were as follows: Forward, TGACTT CAACAGCGACACCCA and reverse, CACCCTGTTGCT GTAGCCAAA. The relative mRNA expression levels were determined by the cycle threshold $(\mathrm{Ct})$ normalized to GAPDH expression, using the $2^{-\Delta \Delta \mathrm{Cq}}$ formula (18).

Western blot analysis. The knockdown efficiency of SCIN shRNA in BC cells transfected with GV115-SCIN shRNA or control shRNA LV was determined. At 2 days post-LV transfection, the cells were collected and lysed in radioimmunoprecipitation lysis buffer (BioTeke Corporation, Beijing, China). Protein concentration was measured using the BCA Protein assay kit (Beyotime Institute of Biotechnology, Haimen, China), and $20 \mu \mathrm{g}$ protein lysates in each pane were separated by $10 \%$ SDS-PAGE, followed by transfer to polyvinylidene difluoride (PVDF) membranes (EMD Millipore, Billerica, MA, USA). Subsequently, the PVDF membranes were blocked in 5\% skimmed milk diluted with TBST (cat no. BD232100, Solarbio Co, Beijing, China) for $1 \mathrm{~h}$ at room temperature. The membranes were then incubated with primary rabbit anti-SCIN monoclonal antibody (mAb) (80 kDa, 1:200; cat no. HPA020518, Sigma-Aldrich; Merck KGaA, Darmstadt, Germany) and GAPDH mouse mAb (37 kDa, 1:1,000; cat no. SC-32233, Santa Cruz Biotechnology, Dallas TX) at $4^{\circ} \mathrm{C}$ overnight. Thereafter, the membranes were incubated with secondary goat anti-rabbit IgG (1:2,000; cat. no. sc-2004, Santa Cruz Biotechnology, Inc.) and goat anti-mouse IgG (1:2,000; cat. no. sc-2005, Santa Cruz Biotechnology, Inc.) antibodies for $1.5 \mathrm{~h}$ at room temperature, respectively. The protein bands were visualized using the Pierce ${ }^{\mathrm{TM}}$ ECL Western Blotting Substrate kit (Thermo Fisher Scientific, Inc.).

Cell Celigo analysis. LV infected MDA-MB-231 and T-47D cells were seeded into 96 -well plates at a concentration of 2,000 cells/well in incubator with $4{ }^{\circ} \mathrm{C}$ and $5 \% \mathrm{CO}_{2}$ for 3 days. The cell nuclei were stained with Hoechst $(2.6 \mu \mathrm{g} / \mathrm{ml}$; Invitrogen; Thermo Fisher Scientific, Inc.) for $5 \mathrm{~min}$ at room temperature to quantify absolute cell number; green fluorescence-tagged shRNA was measured by a fluorescence microscope at x100 magnification to quantify cellular siRNA uptake. All analyses were performed using a Celigo cytometer (Nexcelom Bioscience, Lawrence, MA, USA) over a period of 5 days. Gross quantitative analysis for each fluorescence channel was performed, including total counts of gated events.

3-(4,5-dimethylthiazol-2-yl)-2,5-diphenyltetrazolium bromide (MTT) assay. BC cells were seeded into a 96-well plate (2,000 cells/well) 3 days after LV transfection. The MTT assay was performed per the manufacturer's protocols (cat no. JT343; Gen-view, Beijing, China). Briefly, at the indicated time points $(1,2,3,4$ and 5 days), the MTT solution $(5 \mathrm{mg} / \mathrm{ml})$ was added to the wells and the cells were further incubated at $37^{\circ} \mathrm{C}$ for $4 \mathrm{~h}$. The formazan dye was dissolved with $100 \mu \mathrm{l}$ dimethyl sulfoxide at $37^{\circ} \mathrm{C}$ for $2-5 \mathrm{~min}$. The optical density of the dye was measured using a microplate reader at $490 \mathrm{~nm}$ absorbance and $570 \mathrm{~nm}$ as the wavelength reference.

Caspase-3/7 activity assay. Caspase-3/7 activity was determined using the Caspase-Glo 3 and 7 kit (cat no. G8091, Promega Corporation), according to the manufacturer's protocols, following plating of transfected cells in 96-well plates (in triplicate) for $72 \mathrm{~h}$.

Cell apoptosis. Cell apoptosis was analyzed using the allophycocyanin (APC)/annexin V kit (cat no. 88-8007, eBioscience; Thermo Fisher Scientific, Inc.) according to the manufacturer's protocols. After 5 days of transfection, the cells were stained for $10-15 \mathrm{~min}$ in $100 \mu \mathrm{l}$ cell suspension buffer, containing $5 \mu \mathrm{l}$ annexin $\mathrm{V}$-APC stain, at room temperature in the dark. Cell apoptosis was analyzed by flow cytometry (EMD Millipore).

Statistical analysis. All statistical analyses were performed using SPSS 20.0 (IBM Corp., Armonk, NY, USA). P<0.05 was considered to indicate a statistically significant difference. Association between SCIN expression and clinicopathological features of patients with BC, as well as RT-qPCR experiments, were evaluated using the $\chi^{2}$ test. All other data sets were evaluated using Student's t-test and expressed as the mean \pm standard error of the mean.

\section{Results}

SCIN expression is increased in breast cancer tissues. The IHC results revealed that SCIN protein expression was elevated in BC tissue samples compared with that in control mammary fibroadenoma or fibroadenomatoid hyperplasia tissue samples (Fig. 1). SCIN expression in the cytoplasm of BC ductal epithelial hyperplasia cells was primarily detected with varying intensity (Fig. 1A-D). However, very weak or no SCIN expression was observed in mammary fibroadenoma or fibroadenomatoid hyperplasia tissue samples (Fig. 1E and F). Quantification of SCIN expression in the $\mathrm{BC}$ tissue samples demonstrated that $60.9 \%$ of the BC tissue samples (28/46) revealed high SCIN expression compared with $28.6 \%$ of the mammary fibroadenoma or fibroadenomatoid hyperplasia tissue samples $(6 / 21)(\mathrm{P}=0.014)$ (Table I; Fig. 1).

Further analysis of the association between SCIN expression and clinicopathological features demonstrated no significant differences between high and low expression of SCIN. The proportion of high SCIN-expressing Ki-67-positive $\mathrm{BC}$ tissue samples $(78.6 \%, 22 / 28)$ was higher compared with the Ki-67-negative BC tissue samples (21.4\%, 6/28); however, this difference was not statistically significant (Table II). This observation may indicate that SCIN expression in BC tissue is associated with $\mathrm{Ki}-67$ expression, suggesting that changes in SCIN expression could affect BC cell proliferation. 
Table I. Comparison of SCIN expression in breast fibroadenoma and breast cancer tissues.

\begin{tabular}{lcccrr}
\hline & & \multicolumn{2}{c}{ SCIN expression } & & \\
\cline { 3 - 5 } Type of tissue & $\mathrm{n}$ & None/low, $\mathrm{n}(\%)$ & High, n (\%) & $\chi^{2}$ & P-value \\
\hline Fibroadenoma of the breast & 21 & $15(71.4)$ & $6(28.6)$ & 6.017 & 0.014 \\
Breast cancer & 46 & $18(39.1)$ & $28(60.9)$ & & \\
\hline
\end{tabular}

SCIN, scinderin.

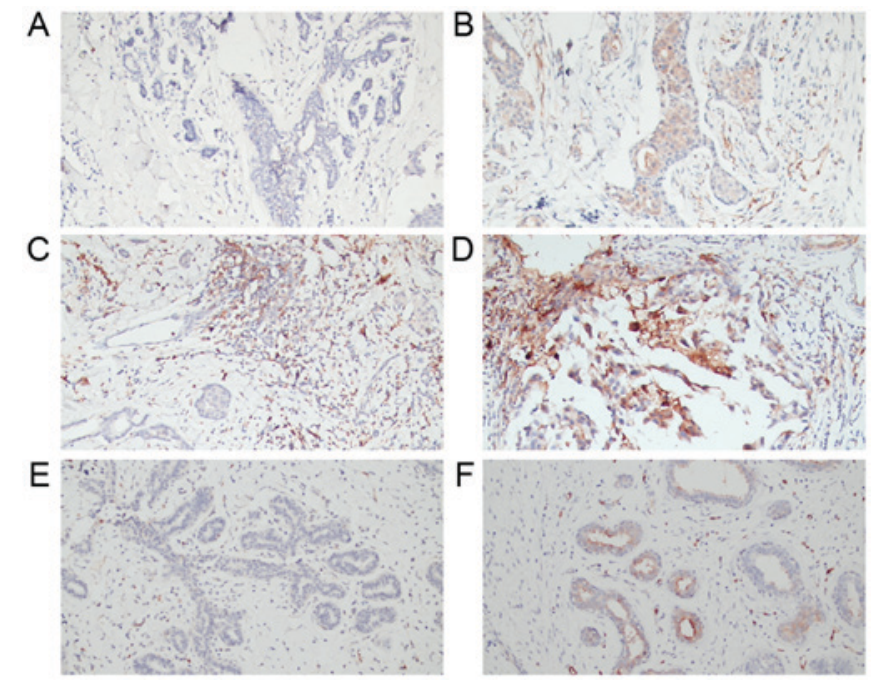

Figure 1. Immunohistochemistry analysis of SCIN expression in fibroadenoma and BC tissue samples. Representative images of (A) negative, (B) weak, (C) moderate and (D) strong SCIN staining in BC tissue, (E) negative and $(\mathrm{F})$ and weak SCIN staining in fibroadenoma (original magnification, x200). SCIN, scinderin; BC, breast cancer.

SCIN is expressed in breast cancer cells. To investigate the functional contribution of SCIN in BC, the Gene Expression Omnibus database was initially utilized (GDS4296, Affymetrix Human Genome U133 Plus 2.0 Array; Affmetrix; Thermo Fisher Scientific, Inc. https://www.ncbi.nlm.nih. gov/geo/tools/profileGraph.cgi?ID=GDS4296\%3A222272_x_ at\&sortby $=$ tissue) to determine SCIN levels in BC samples (19). Using this database, SCIN was revealed to be expressed in multiple BC cell lines, including BT-549, HS578T, MCF-7, MDA-MB-231 and T-47D. As presented in Fig. 2A, SCIN expression was higher in MCF-7 and T-47D cells. SCIN expression in BC cells was further validated using RT-qPCR (Fig. 2B). The MDA-MB-231 cell line is a highly invasive and metastatic, while T-47D and MCF-7 cell lines exhibit comparatively mild invasive and metastatic capabilities. Therefore, MDA-MB-231 and T-47D cells were selected to examine the effect of SCIN on different BC cells.

Suppressed SCIN expression inhibits breast cancer cell proliferation. In order to investigate the molecular function of SCIN in BC cells, SCIN expression was ablated using LV-mediated knockdown. Following 3 days of LV transfection, infection efficiency and good cell morphology were observed by fluorescent microscope with $\mathrm{x} 200$ magnification (Fig. 3A and B).

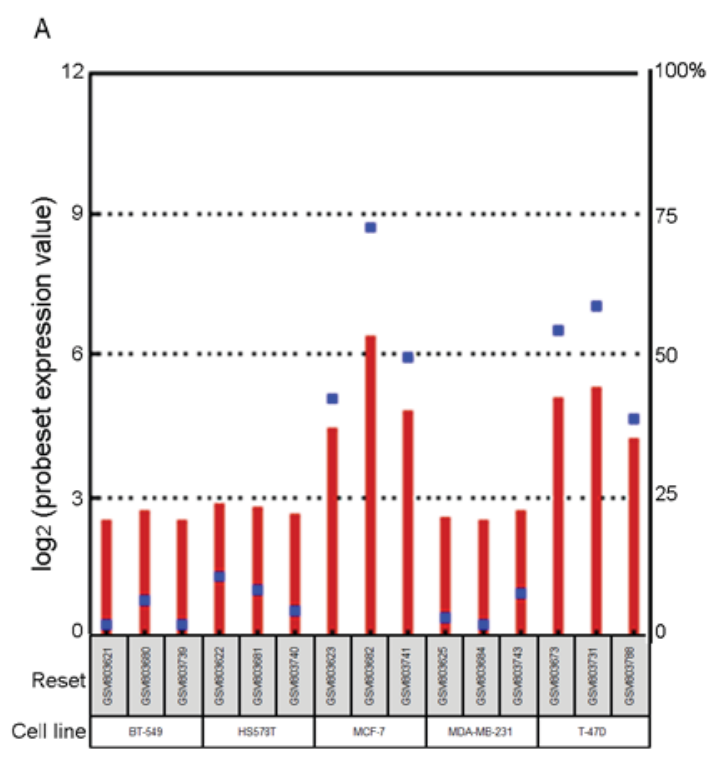

- percentile rank within the sample

B

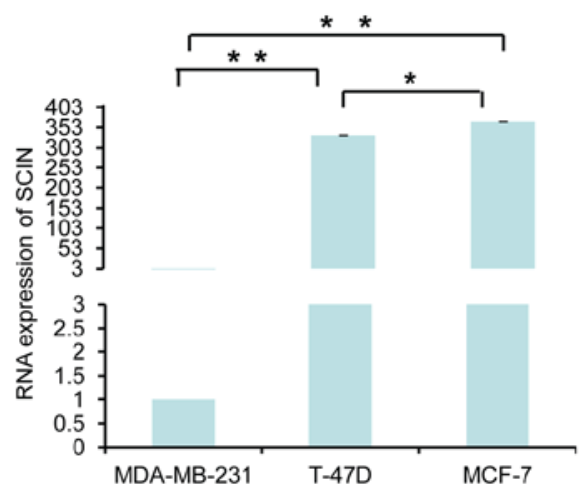

Figure 2. Assessment of SCIN expression using the GEO database and RT-qPCR. (A) Bioinformatics analysis of SCIN expression in five BC cell lines from the GEO database. (B) RT-qPCR analysis of SCIN expression in three BC cell lines; MDA-MB-231, T-47D and MCF-7. GAPDH expression was used as a control to normalize SCIN expression. SCIN, scinderin; BC, breast cancer; RT-qPCR, reverse transcription-quantitative polymerase chain reaction; GEO, Gene Expression Omnibus. ${ }^{* *} \mathrm{P}<0.05,{ }^{*} \mathrm{P}>0.05$.

Then RT-qPCR analysis revealed that SCIN mRNA expression was significantly lower in MDA-MB-231 and T-47D cells in the shSCIN group compared with that in the shCtrl group (Fig. 3C and D). Similarly, a reduction in SCIN protein expression was confirmed in BC cells that were transfected with shSCIN (Fig. 3E and F). 
Table II. Association between SCIN expression and clinicopathological features in patients with breast cancer.

\begin{tabular}{|c|c|c|c|c|c|}
\hline \multirow[b]{2}{*}{ Pathological variables } & \multirow[b]{2}{*}{$\mathrm{n}$} & \multicolumn{2}{|c|}{ SCIN expression } & \multirow[b]{2}{*}{$\chi^{2}$} & \multirow[b]{2}{*}{ P-value } \\
\hline & & None/low, n (\%) & High, n (\%) & & \\
\hline Pathological type & & & & 0.634 & 0.728 \\
\hline IDC & 36 & $13(36.1)$ & $23(63.9)$ & & \\
\hline DCIS & 6 & $3(50.0)$ & $3(50.0)$ & & \\
\hline Mucoid carcinoma & 4 & $2(50.0)$ & $2(50.0)$ & & \\
\hline ER & & & & 0.417 & 0.518 \\
\hline Negative & 18 & $6(33.3)$ & $12(66.7)$ & & \\
\hline Positive & 28 & $12(42.9)$ & $16(57.1)$ & & \\
\hline PR & & & & 0.225 & 0.635 \\
\hline Negative & 25 & $9(36.0)$ & $16(64.0)$ & & \\
\hline Positive & 21 & $9(42.9)$ & $12(57.1)$ & & \\
\hline HER2 & & & & 0.004 & 0.949 \\
\hline Negative & 36 & $14(38.9)$ & $22(61.1)$ & & \\
\hline Positive & 10 & $4(40.0)$ & $6(60.0)$ & & \\
\hline Ki-67 & & & & 0.004 & 0.949 \\
\hline Negative & 10 & $4(40.0)$ & $6(60.0)$ & & \\
\hline Positive & 36 & $14(38.9)$ & $22(61.1)$ & & \\
\hline Stage & & & & 0.48 & 0.488 \\
\hline$\leq \mathrm{II}$ & 38 & $14(36.8)$ & $24(63.2)$ & & \\
\hline$\geq \mathrm{III}$ & 8 & $4(50.0)$ & $4(50.0)$ & & \\
\hline Molecular characteristics & & & & 4.036 & 0.258 \\
\hline Luminal A & 7 & $5(71.4)$ & $2(28.6)$ & & \\
\hline Luminal B & 21 & $7(33.3)$ & $14(66.7)$ & & \\
\hline HER2 overexpression & 10 & $4(40.0)$ & $6(60.0)$ & & \\
\hline Triple-negative & 8 & $2(25.0)$ & $6(75.0)$ & & \\
\hline
\end{tabular}

Ki-67 expression: $\leq 14 \%, \mathrm{Ki}-67$ negative staining; and $\geq 15 \%$, Ki-67 positive staining. IDC, invasive ductal carcinoma; DCIS, ductal carcinoma in situ; SCIN, scinderin; HER2, human epidermal growth factor receptor 2; ER, estrogen receptor; PR, progesterone receptor.

Next, proliferation was assessed in MDA-MB-231 and T-47D cells using the in vitro Celigo assay 3 days after shSCIN or shCtrl transfection. A marked reduction in proliferation of MDA-MB-231 and T-47D cells was identified (Fig. 3G-L). A separate experiment using the MTT assay confirmed that cellular metabolic activity was decreased in the MDA-MB-231 when silencing SCIN expression (Fig. 3M and N). The lower cellular metabolic activity was also examined in the T-47D cells with knockdown of SCIN expression (Fig. 30 and P). Thus, these results indicate that SCIN-knockdown inhibits BC cell proliferation and cellular metabolic activity.

SCIN-knockdown induces cell apoptosis. To further investigate the mechanism by which SCIN-knockdown reduces cell proliferation, Caspase-3/7 activity in MDA-MB-231 and T-47D cells was analyzed following $72 \mathrm{~h}$ of LV transfection of shSCIN and shCtrl. SCIN-knockdown significantly enhanced Caspase-3/7 activity in the two cell types $(\mathrm{P}<0.01$; Fig. $4 \mathrm{~A}$ and $\mathrm{B})$. Flow cytometric analysis of cell apoptosis was also performed. As presented in Fig. 4C-F, apoptosis was significantly increased in the MDA-MB-231 and T-47D cells in the shSCIN group $(\mathrm{P}<0.01)$ following 5 days of $\mathrm{LV}$ transfection.

\section{Discussion}

The majority of cases of BC-associated mortality occur due to metastasis, for which cell proliferation is the biggest contributor. Cell proliferation is directly and indirectly associated with $\mathrm{BC}$ prognosis (20). Multiple studies evaluating the role of numerous proliferation genes have added to the current understanding of BC metastasis (21-23), but the functional contribution of SCIN expression in $\mathrm{BC}$ proliferation has been relatively under-reported. Thus, in the present study, the role of SCIN in BC proliferation and apoptosis was investigated. The results demonstrated that SCIN expression is increased in BC cells at the protein (IHC) and mRNA (RT-qPCR) levels. Furthermore, knockdown of SCIN expression in MDA-MB-231 and T-47D cells, with LV-mediated gene silencing technology, suggested that SCIN expression regulates cell proliferation and apoptosis. Inhibition of proliferation and induction of apoptosis were observed in MDA-MB-231 and T-47D cells following SCIN-knockdown.

Uncontrolled cell proliferation is a central hallmark of all types of cancer. Since the actin cytoskeleton serves a prominent role in cancer cell cycle regulation (24), a number of cytoskeleton-associated proteins have been studied in reference 

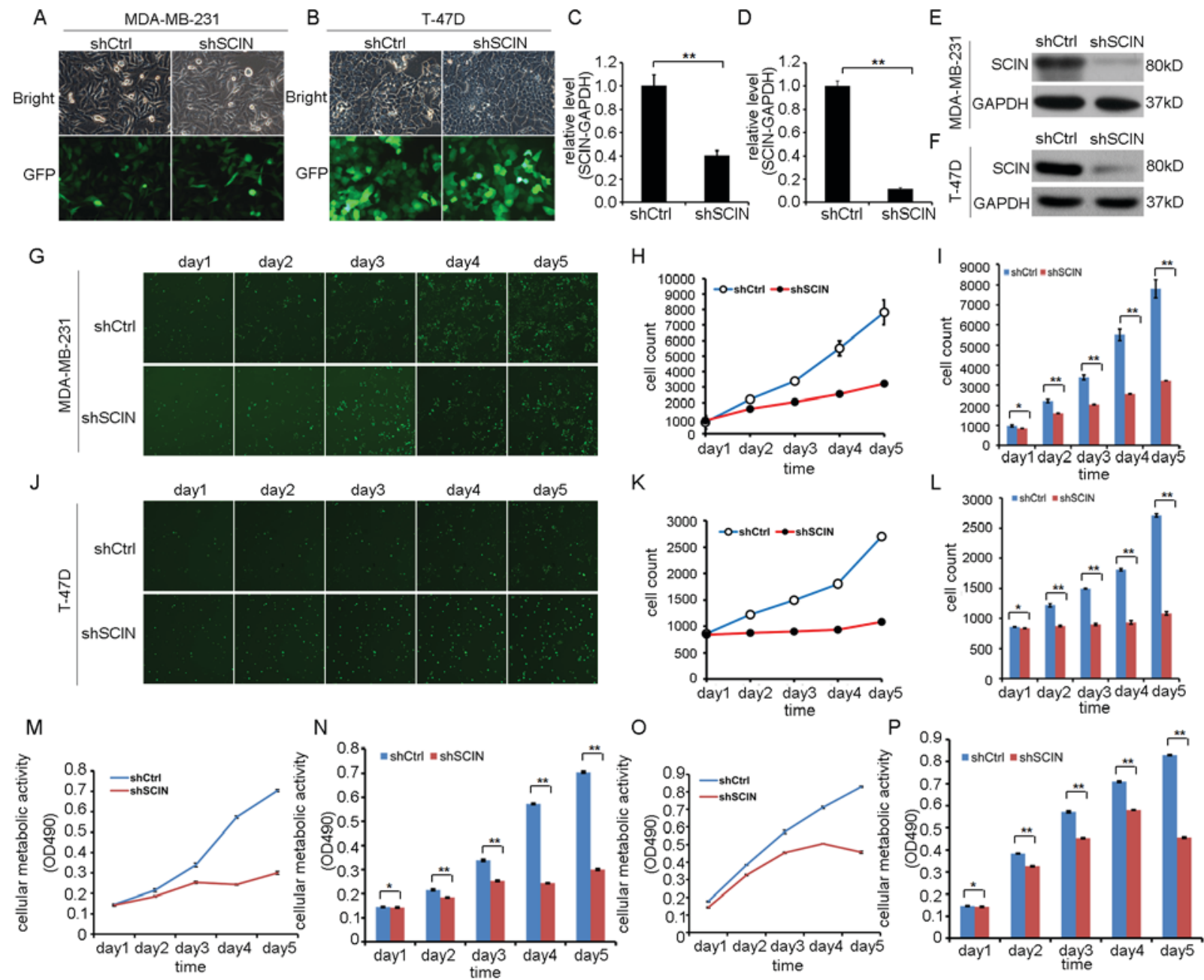

Figure 3. Knockdown of SCIN expression by lentivirus-mediated shRNA and its effect on cell proliferation. Representative images of SCIN-knockdown in (A) MDA-MB-231 and (B) T-47D cells, assessed by GFP expression using fluorescence microscopy (x200 magnification). SCIN RNA expression following knockdown in (C) MDA-MB-231 and (D) T-47D cells, assessed by reverse transcription-quantitative polymerase chain reaction analysis. GAPDH expression was used as an internal control. Data are presented as mean values \pm standard error of the mean of three independent experiments. ${ }^{* *} \mathrm{P}<0.01$ compared with the shCtrl group. Western blot analysis of SCIN-knockdown in (E) MDA-MB-231 and (F) T-47D cells. Representative blots of three separate experiments. (G) Representative images of Celigo assay (x100 magnification), (H) cumulative data of Celigo assay, and (I) bar graphs indicate the mean cell count values in MDA-MB-231 cells transfected with shCtrl or shSCIN were illustrated. (J) Representative images of Celigo assay (x100 magnification), (K) cumulative data of Celigo assay, and (L) bar graphs indicate the mean cell count values in T-47D cells transfected with shCtrl or shSCIN were presented. Cellular metabolic activity, assessed with MTT assay, of (M) MDA-MB-231 and (O) T-47D cells. Bar graphs indicate the mean cellular metabolic activity values in the MDA-MB-231 (N) and T-47D cells (P). All data represented are mean values \pm standard error of the mean from four independent experiments. Student's t-test was used for statistical analysis. ${ }^{* *} \mathrm{P}<0.01$. ${ }^{*} \mathrm{P}>0.05$ vs. control, SCIN, scinderin; BC, breast cancer; shCtrl, short hairpin control; GFP, green fluorescent protein; OD, optical density.

to BC (25-27). SCIN contains six gelsolin-like domains, three actin-binding sites and two calcium-binding sites $(6,28)$. Previous studies have linked abnormal SCIN expression to various carcinomas, as well as to cell proliferation. In the present study, SCIN expression was revealed to be associated with Ki-67-positive $\mathrm{BC}$ cells in tissue samples. Although not significant, this trend does indicate that there may be an association between SCIN and Ki-67 expression in BC tissues. Since Ki-67 is a cellular marker of proliferation, the results of the current study indicate that SCIN may also be involved in the proliferation of BC cells. Consistent with this conclusion, SCIN-knockdown was revealed to inhibit breast carcinoma cell proliferation in vitro in the present study. This observation was in accordance with studies by Liu et al (10) and Wang et al (9), who revealed that SCIN overexpression enhanced human lung and prostate carcinoma cell proliferation. Therefore, as SCIN governs filamentous actin
(F-actin) cytoskeleton remodeling, it is possible that SCIN's effects on cell proliferation may be mediated through F-actin. This hypothesis requires further analysis, for example, observing whether the F-actin cytoskeleton is changed when SCIN expression is silenced in BC cells. Further, animal experiments should be performed to validate the proliferative ability of SCIN.

Apoptosis can significantly contribute to normal cell physiology. Apoptosis maintains absolute cell numbers, affects wound healing and regulates remodeling (29). Abnormalities in apoptosis lead to numerous diseases, including cancer, in which the normal mechanisms of cell cycle regulation become dysfunctional (30). Inducing apoptosis during carcinogenesis can contribute to the treatment and prevention of cancer development and progression $(31,32)$. In the current study, SCIN-knockdown induced cell apoptosis. Therefore SCIN may serve an important role in the development and progression of $\mathrm{BC}$. 
A

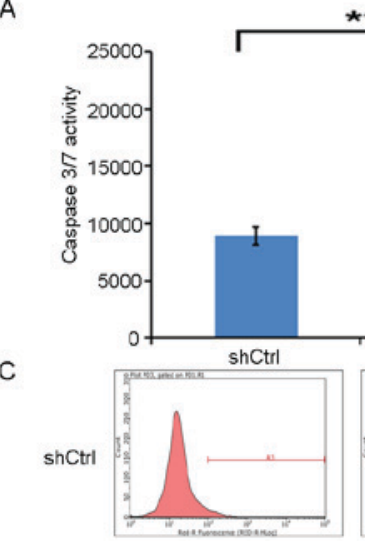

ANNEXIN-V

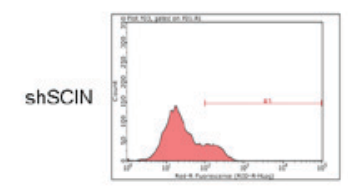

ANNEXIN-V

E

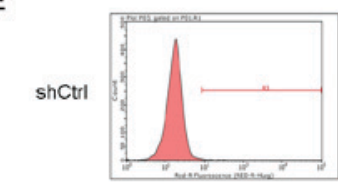

ANNEXIN-V

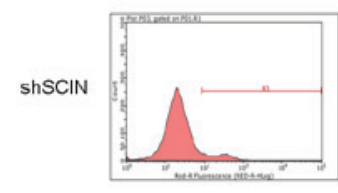

ANNEXIN-V
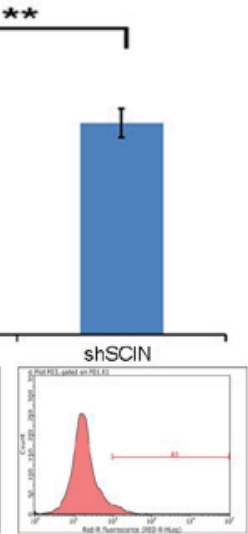

ANNEXIN-V

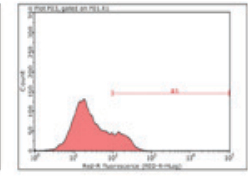

ANNEXIN-V

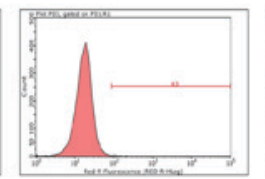

ANNEXIN-V

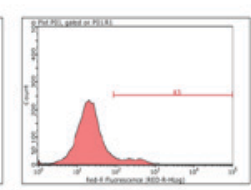

ANNEXIN-V
B
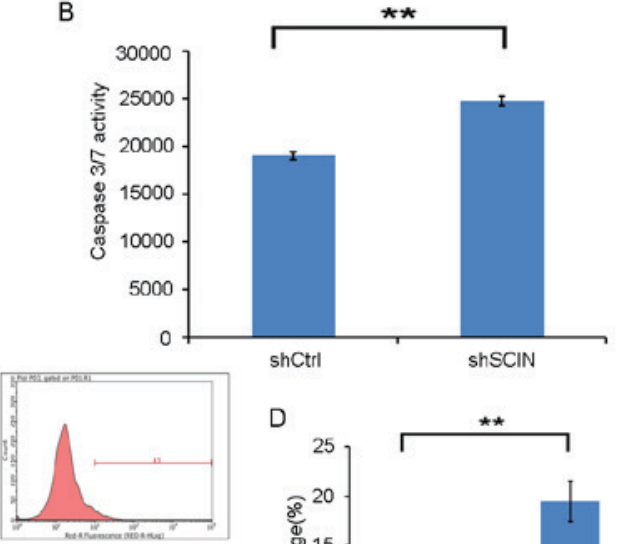

ANNEXIN-V

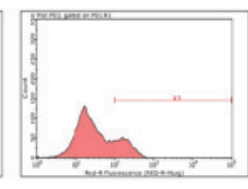

ANNEXIN-V

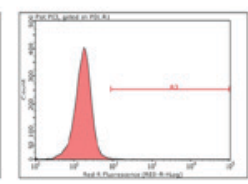

ANNEXIN-V

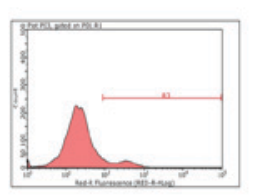

ANNEXIN-V

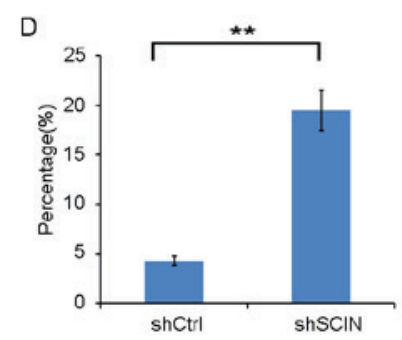

F

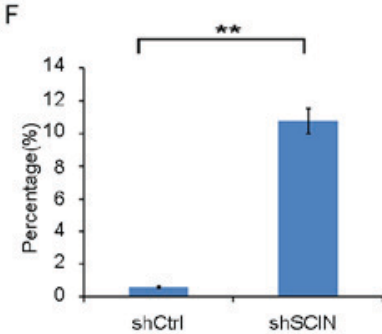

Figure 4. Effects of SCIN-knockdown on cell apoptosis. Caspase 3/7 activity in (A) MDA-MB-231 and (B) T-47D cells 3 days following shRNA transfection. (C) Cumulative data of annexin-V staining analyzed by flow cytometry in MDA-MB-231 cells transfected with shCtrl or shSCIN and (D) its representative histograms were presented. (E) Cumulative data of annexin-V staining analyzed by flow cytometry in T-47D cells transfected with shCtrl or shSCIN and (F) its representative histograms were presented. All data are presented as mean values \pm standard error of the mean from four independent experiments. Student's t-test was used for statistical analysis. ${ }^{* *} \mathrm{P}<0.01$. shCtrl, short hairpin control; SCIN, scinderin.

In summary, the results of the current study demonstrated that shRNA-mediated SCIN-knockdown inhibited breast cancer cell proliferation and induced apoptosis. This sheds new light on the novel role of SCIN in BC development and progression.

\section{Acknowledgements}

Not applicable

\section{Funding}

This study was funded by the following grants: The Science, Technology and Innovation Committee of Shenzhen Municipality (grant no. JCYJ20160425103015129) and the Science, Technology and Innovation Committee of Shenzhen Municipality (grant no. JSGG20160226202029158).

\section{Availability of data and materials}

All data used during the current study are available from the corresponding author on reasonable request.

\section{Authors' contributions}

WJ designed the study and wrote the manuscript. XZ, JW and YL performed the experiments. $\mathrm{CH}$ analyzed data. XW and RL also contributed to study design, data analysis and modified the manuscript.

\section{Ethics approval and consent to participate}

This study was approved by the Ethics Committee of The Second People's Hospital of Shenzhen, and written consent was obtained from all study participants.

\section{Patient consent for publication}

Patients provided written consent for the publication of data and any associated images.

\section{Competing interests}

The authors declare that they have no competing interests. 


\section{References}

1. Harbeck N and Gnant M: Breast cancer. Lancet 389: 1134-1150, 2017.

2. Torre LA, Bray F, Siegel RL, Ferlay J, Lortet-Tieulent J and Jemal A: Global cancer statistics, 2012. CA Cancer J Clin 65: 87-108, 2015.

3. Razzaghi H, Quesnel-Crooks S, Sherman R, Joseph R, Kohler B, Andall-Brereton G, Ivey MA, Edwards BK, Mery L, Gawryszewski V and Saraiya M: Leading causes of cancer mortality-Caribbean Region, 2003-2013. Morb Mortal Wkly Rep 65:1395-1400,2016.

4. Lejen T, Pene TD, Rosé SD and Trifaró JM: The role of different Scinderin domains in the control of F-actin cytoskeleton during exocytosis. Ann N Y Acad Sci 971: 248-250, 2002.

5. Chen XM, Guo JM, Chen P, Mao LG, Feng WY, Le DH and Li KQ: Suppression of scinderin modulates epithelialmesenchymal transition markers in highly metastatic gastric cancer cell line SGC7901. Mol Med Rep 10: 2327-2333, 2014.

6. Rodriguez Del Castillo A, Lemaire S, Tchakarov L, Jeyapragasan M, Doucet JP, Vitale ML and Trifaró JM: Chromaffin cell scinderin, a novel calcium-dependent actin filament-severing protein. EMBO J 9: 43-52, 1990.

7. Lueck A, Brown D and Kwiatkowski DJ: The actin-binding proteins adseverin and gelsolin are both highly expressed but differentially localized in kidney and intestine. J Cell Sci 111 3633-3643, 1998.

8. Tchakarov L, Vitale ML, Jeyapragasan M, Rodriguez Del Castillo A and Trifaró JM: Expression of scinderin, an actin filament-severing protein, in different tissues. FEBS Lett 268: 209-212, 1990

9. Wang D, Sun SQ, Yu YH, Wu WZ, Yang SL and Tan JM: Suppression of SCIN inhibits human prostate cancer cell proliferation and induces G0/G1 phase arrest. Int J Oncol 44: 161-166, 2014.

10. Liu H, Shi D, Liu T, Yu Z and Zhou C: Lentivirus-mediated silencing of SCIN inhibits proliferation of human lung carcinoma cells. Gene 554: 32-39, 2015.

11. Zunino R, Li Q, Rosé SD, Romero-Benítez MM, Lejen T, Brandan NC and Trifaró JM: Expression of scinderin in megakaryoblastic leukemia cells induces differentiation, maturation, and apoptosis with release of plateletlike particles and inhibits proliferation and tumorigenesis. Blood 98: 2210-2219, 2001.

12. Liu JJ, Liu JY, Chen J, Wu YX, Yan P, Ji CD, Wang YX, Xiang DF, Zhang X, Zhang P, et al: Scinderin promotes the invasion and metastasis of gastric cancer cells and predicts the outcome of patients. Cancer Lett 376: 110-117, 2016.

13. Coratti A, Fernandes E, Lombardi A, Di Marino M Annecchiarico M, Felicioni L and Giulianotti PC: Robot-assisted surgery for gastric carcinoma: Five years follow-up and beyond A single western center experience and long-term oncological outcomes. Eur J Surg Oncol 41: 1106-1113, 2015.

14. Lin H, Huang JF, Qiu JR, Zhang HL, Tang XJ, Li H, Wang CJ, Wang ZC, Feng ZQ and Zhu J: Significantly upregulated TACSTD2 and Cyclin D1 correlate with poor prognosis of invasive ductal breast cancer. Exp Mol Pathol 94: 73-78, 2013.

15. Shi H, Chen S, Jin H, Xu C, Dong G, Zhao Q, Wang W, Zhang H, Lin W, Zhang J, et al: Downregulation of MSP58 inhibits growth of human colorectal cancer cells via regulation of the cyclin D1-cyclin-dependent kinase 4-p21 pathway. Cancer Sci 100: 1585-1590, 2009.
16. Camp RL, Dolled-Filhart M and Rimm DL: X-tile: A new bio-informatics tool for biomarker assessment and outcome-based cut-point optimization. Clin Cancer Res 10: 7252-7259, 2004.

17. Cheang MC, Chia SK, Voduc D, Gao D, Leung S, Snider J, Watson M, Davies S, Bernard PS, Parker JS, et al: Ki67 index, HER2 status, and prognosis of patients with luminal B breast cancer. J Natl Cancer Inst 101: 736-750, 2009.

18. Livak KJ and Schmittgen TD: Analysis of relative gene expression data using real-time quantitative PCR and the 2(-Delta Delta (CT)) method. Methods 25: 402-408, 2001.

19. Shankavaram UT, Reinhold WC, Nishizuka S, Major S, Morita D, Chary KK, Reimers MA, Scherf U, Kahn A, Dolginow D, et al: Transcript and protein expression profiles of the NCI-60 cancer cell panel: An integromic microarray study. Mol Cancer Ther 6: 820-832, 2007.

20. van Diest PJ, van der Wall E and Baak JP: Prognostic value of proliferation in invasive breast cancer: A review. J Clin Pathol 57: 675-681, 2004

21. Kim K, Son MY, Jung CR, Kim DS and Cho HS: EHMT2 is a metastasis regulator in breast cancer. Biochem Biophys Res Commun 496:758-762, 2018.

22. Song L, Liu D, Zhao Y, He J, Kang H, Dai Z, Wang X, Zhang S, Zan Y and Xue X: Sinomenine reduces growth and metastasis of breast cancer cells and improves the survival of tumor-bearing mice through suppressing the SHh pathway. Biomed Pharmacother 98: 687-693, 2018.

23. Gao Y, Li F, Zhou H, Yang Y, Wu R, Chen Y, Li W, Li Y, Xu X, $\mathrm{Ke} \mathrm{C}$ and Pei Z: Down-regulation of MRPS23 inhibits rat breast cancer proliferation and metastasis. Oncotarget 8: 71772-71781, 2017.

24. Hall A: The cytoskeleton and cancer. Cancer Metastasis Rev 28: 5-14, 2009.

25. Kas SM, de Ruiter JR, Schipper K, Annunziato S, Schut E, Klarenbeek S, Drenth AP, van der Burg E, Klijn C, Ten Hoeve JJ, et al: Insertional mutagenesis identifies drivers of a novel oncogenic pathway in invasive lobular breast carcinoma. Nat Genet 49: 1219-1230, 2017.

26. Mercado-Matos J, Clark JL, Piper AJ, Janusis J and Shaw LM: Differential involvement of the microtubule cytoskeleton in insulin receptor substrate 1 (IRS-1) and IRS-2 signaling to AKT determines the response to microtubule disruption in breast carcinoma cells. J Biol Chem 292: 7806-7816, 2017.

27. Kumar N, Hati S, Munshi P, Sen S, Sehrawat S and Singh S: A novel spiroindoline targets cell cycle and migration via modulation of microtubule cytoskeleton. Mol Cell Biochem 429: 11-21, 2017.

28. Chumnarnsilpa S, Lee WL, Nag S, Kannan B, Larsson M, Burtnick LD and Robinson RC: The crystal structure of the C-terminus of adseverin reveals the actin-binding interface. Proc Natl Acad Sci USA 106: 13719-13724, 2009.

29. Elmore S: Apoptosis: A review of programmed cell death. Toxicol Pathol 35: 495-516, 2007.

30. King KL and Cidlowski JA: Cell cycle regulation and apoptosis. Annu Rev Physiol 60: 601-617, 1998.

31. Jordan VC: The new biology of estrogen-induced apoptosis applied to treat and prevent breast cancer. Endocr Relat Cancer 22: R1-R31, 2015.

32. Carew JS, Espitia CM,Zhao W, Kelly KR, Coffey M, Freeman JW and Nawrocki ST: Reolysin is a novel reovirus-based agent that induces endoplasmic reticular stress-mediated apoptosis in pancreatic cancer. Cell Death Dis 4: e728, 2013. 